\title{
CCL3L1 copy number, HIV load, and immune reconstitution in sub-Saharan Africans
}

\author{
Eleni Aklillu', Linda Odenthal-Hesse ${ }^{2}$, Jennifer Bowdrey ${ }^{2}$, Abiy Habtewold ${ }^{1,3}$, Eliford Ngaimisi ${ }^{1,4}$, Getnet Yimer ${ }^{1,3}$, \\ Wondwossen Amogne ${ }^{5,6}$, Sabina Mugusi ${ }^{7}$, Omary Minzi ${ }^{4}$, Eyasu Makonnen ${ }^{3}$, Mohammed Janabi ${ }^{8}$, \\ Ferdinand Mugusi $i^{8}$, Getachew Aderaye ${ }^{5}$, Robert Hardwick ${ }^{2}$, Beiyuan $\mathrm{Fu}^{9}$, Maria Viskaduraki ${ }^{10}$, Fengtang Yang ${ }^{9}$ \\ and Edward J Hollox ${ }^{2 *}$
}

\begin{abstract}
Background: The role of copy number variation of the CCL3L1 gene, encoding MIP1a, in contributing to the host variation in susceptibility and response to HIV infection is controversial. Here we analyse a sub-Saharan African cohort from Tanzania and Ethiopia, two countries with a high prevalence of HIV-1 and a high co-morbidity of HIV with tuberculosis.

Methods: We use a form of quantitative PCR called the paralogue ratio test to determine CCL3L1 gene copy number in 1134 individuals and validate our copy number typing using array comparative genomic hybridisation and fiber-FISH.

Results: We find no significant association of CCL3L1 gene copy number with HIV load in antiretroviral-naïve patients prior to initiation of combination highly active anti-retroviral therapy. However, we find a significant association of low CCL3L1 gene copy number with improved immune reconstitution following initiation of highly active anti-retroviral therapy $(p=0.012)$, replicating a previous study.
\end{abstract}

Conclusions: Our work supports a role for CCL3L1 copy number in immune reconstitution following antiretroviral therapy in HIV, and suggests that the MIP1a -CCR5 axis might be targeted to aid immune reconstitution.

\section{Background}

AIDS, caused by the retrovirus HIV, is predicted by 2030 to become globally the single largest cause of morbidity, as measured by disability-adjusted life-years [1]. African countries currently have the highest disease burden of HIV, with 9.2\% prevalence in Addis Ababa in Ethiopia and over $10 \%$ in Dar-es-Salaam in Tanzania, yet almost all genetic studies have focused on cohorts from Western countries [2]. The genetic architecture of HIV susceptibility in Africans is likely to be different to Europeans, yet genome-wide association studies of host susceptibility to HIV have not yielded any significant results [3]. These studies miss regions that show copy number variation, particularly structurally complex

\footnotetext{
* Correspondence: Ejh33@le.ac.uk

${ }^{2}$ Department of Genetics, University of Leicester, University Road, LE1 7RH, Leicester, UK

Full list of author information is available at the end of the article
}

regions that are not correlated with alleles at flanking SNP markers [4].

Copy number variation (CNV) is defined as the variation in copy number of a given DNA sequence in a diploid genome. CNV is common in the genome, affects gene expression, and involves immune response genes [5-7], suggesting that it may affect susceptibility of the host to infectious disease. CNV of the killer cell immunoglobulin receptor genes has been shown to affect host control of HIV infection, as determined by the viral load (VL) at setpoint [8], and we have recently shown association of $\beta$-defensin $\mathrm{CNV}$ both with HIV viral load at initiation of highly-active anti-retroviral therapy (HAART) and with consequent immune reconstitution [9].

The genes CCL3L1/CCL4L1 encode the chemokines MIP- $1 \alpha$ and MIP- $1 \beta$ which are both ligands for the chemokine receptor CCR5 used as a co-receptor by $\mathrm{R} 5$ strains of HIV. These genes show CNV, and this has been shown to affect HIV acquisition, progression to

\section{Biomed Central}

(C) 2013 Aklillu et al.; licensee BioMed Central Ltd. This is an open access article distributed under the terms of the Creative Commons Attribution License (http://creativecommons.org/licenses/by/2.0), which permits unrestricted use, distribution, and reproduction in any medium, provided the original work is properly cited. 
AIDS, and immune reconstitution following highly active anti-retroviral therapy (HAART) [10-12]. An attractive model is that these chemokines and HIV compete for the same receptor CCR5, and that increasing copy number increases the levels of chemokine, thereby increasing competition with HIV for the receptor [13]. A gene dosage effect linking gene copy number and protein levels is needed to support this hypothesis, and evidence has been contradictory. Early studies supported a gene dosage effect $[10,11]$, but recent studies have suggested that the influence of extra gene copies on total protein levels is low $[14,15]$. A problem in these experiments is that the protein product of CCL3 (called MIP1 $\alpha-\mathrm{LD} 78 \alpha$ ) and CCL3L1 (MIP1 $\alpha$-LD78 $\beta$ ) cannot be discriminated using standard antibodies. Thus analyses using antibody-based detection of protein products may not detect a gene dosage effect, particularly given the higher levels of CCL3 transcription and presumably MIP $1 \alpha-L D 78 \alpha$ in the blood. Although both protein isoforms signal through CCR5, only the LD78 $\beta$ isoform can be cleaved by dipetidyl peptidase IV to generate a monocyte attractant and CCR1 agonist [16,17]. Indeed, functional evidence remains supportive: measuring the chemotactic response of cells to supernatants from lipopolysaccharide-stimulated monocytes from different individuals supports an effect of different CCL3L1 gene copy number [10]. However, other mechanisms for an effect of CCL3L1 copy number can be invisaged, either directly or indirectly by affecting other immunological phenotypes such as the CD4+ cell count.

Attempts at replicating the genetic association of CCL3L1 copy number and HIV susceptibility have yielded contrasting results. A meta-analysis of nine studies has supported an association of lower CCL3L1 with susceptibility to HIV [18], but this study did not critically analyse the quality of the published data used in the meta-analysis. For example, the use of quantitative PCR to determine CCL3L1 copy number may generate false-positive associations [19-21]. It may be that CCL3L1 and CCLAL1 do not always vary in copy number as a block, which might explain at least some of the heterogeneity in results when different methods are used to determine copy number. However, when more robust reliable methods are applied to large European cohorts there is no evidence of this, suggesting that when measured with sufficient precision and accuracy, CCL3L1 and CCL4L1 covary as a block [22,23]. In common with most of the literature, we refer to this copy number variation as CCL3L1 copy number variation, but it should be remembered that it also involves CCLAL1 and possibly TBC1D3.

CCL3L1 CNV has also been associated with a variety of other infectious diseases, including tuberculosis [24], hepatitis B [25], hepatitis C [26] and Kawasaki Disease [27]. Such association studies are almost always small, use qPCR to type copy number, not necessarily replicated [28], and in some cases the reported association is seen only on a background of a particular genotype at another locus. While such studies are based on reasonable hypotheses concerning the function and interaction of proteins and pathogens, the marginal significance levels and limited power of such studies means that drawing definitive conclusions regarding the role of genetic variation remains difficult. In the most technically- and genetically-thorough study to date, a weak suggestive association with protection from anemia in malarial infection was found, but this family-based study too lacked power to detect anything but strong effects [29].

Evidence from other African studies of CCL3L1 and HIV has been contradictory. In a small Zimbabwean longitudinal cohort, no association of CCL3L1 copy number with HIV status or progression was found [30]. However, analysis of mother-to-child transmission in South Africa suggested that higher copy number was protective against HIV transmission [31]. In this context, we decided to analyse our previously described cohort of HIV patients from Ethiopia and Tanzania for association of CCL3L1 copy number with viral load immediately prior to HAART and immune reconstitution during HAART. African populations are known to have a higher average copy number than European populations $[11,31]$, due either to natural selection or genetic drift. This has the advantage, in an association study context, of providing a wider range of copy number and therefore a potentially larger gene dosage effect. However, there are significant technical challenges in accurately typing multiallelic copy numbers at this, or indeed other, loci. We decided to use the paralogue ratio test (PRT) to determine copy number, which is the most robust technique available for typing this locus on large cohorts $[19,21]$.

\section{Methods \\ Sample collection}

Patient sample, DNA extraction and clinical data collection was as previously described $[9,32,33]$. The study protocol was approved by the Institutional Review Board at the Faculty of Medicine, Addis Ababa University and Ethiopian Science and Technology Ministry; the regional ethical review board in Stockholm at the Karolinska Institutet and the ethical review committee of Muhimbili University of Health and Allied Sciences. Written informed consent was obtained from each subject before the start of this study. DNA samples from the HapMap YRI population (Yoruba from Ibadan, Nigeria) were obtained from Coriell Cell Repositories (Camden, NJ, USA).

The CCR5 $\delta 32$ allele, associated with protection from HIV infection and disease, progression, was not detected in either the Tanzanian or Ethiopian samples [9], where the deletion allele is known to be protective against HIV progression. Patient numbers used at each stage of the 
study are given in Additional file 1: Table S1. Baseline characteristics of patients are given in Additional file 2: Table S2.

\section{Copy number typing}

Copy number typing was performed using the PRT approach described previously [22]. Briefly, data from three separate PRT assays measuring copy number across the CCL3L1 segmental duplication are normalised using four known positive control samples (C0075 - 1 copy, C0150 - 2 copies, C0007 - 3 copies, C0877 - 4 copies), available as part of the human reference control plate HRC-1 from the Health Protection Agency, Porton, UK, analysed with every experimental PCR plate, and then averaged to give an unrounded estimate of copy number. The replicate testing of the four positive controls using the three separate PRT assays produces datapoints that clearly cluster, with clusters showing a linear relationship with copy number, and no assay-specific biases in clustering (Additional file 3: Figure S1).

Samples were tested in duplicate if the coefficient of variation of the values from the three separate assays exceeded a given threshold, typically 0.2 , and the result that gave the lower coefficient of variation taken on to the next stage of analysis. A small number of samples gave consistently high $(>0.5)$ coefficient of variation scores even after repeated testing. This was due to altered copy number of either LTR16 or CCL4, and for these the copy number from the two consensus PRTs was taken forward. However, in general, raw copy number estimates from each of the three PRT assays was highly concordant across samples, with clustering about integer copy numbers evident at lower copy numbers (Additional file: 4 Figure S2).

Integer copy numbers were inferred from mean unrounded copy number estimates using a Gaussian mixture model, implemented in the statistical language $\mathrm{R}$ (package CNVtools [34]). The appropriateness of using Gaussian distributions to model PRT data can be tested by analysis of the data from the positive control samples analysed on every PCR plate. When normalised by copy number, it is clear that the combined dataset fit the Gaussian distribution well, although a number of outliers are seen (Additional file 5: Figure S3a). These outliers seem to be more likely for the lower copy number samples, suggesting that the assumption of the Gaussian distribution not only holds but may even be a stronger assumption for PRTs measuring higher copy numbers (Additional file 5: Figure S3b). A mixture model of nine components was fitted, based on observation of the data and prior studies. The model of variance components was fixed to have similar variance, an assumption supported by the similar variance of the repeated PRT values from the four positive controls. The resulting clustering quality score (Q) was 3.9. A posterior probability of the integer copy number call being correct was given for each sample. Where this probability was below 0.8 , and the probability of the copy number one higher or one lower was therefore $>0.2$, then the mean of a duplicate test (if carried out) was used to call the correct integer copy number.

\section{Fibre FISH}

Fibre-FISH was performed as described previously [35]. Briefly, stretched DNA fibers were prepared from lymphoblastoid cell lines. Fosmid DNA was prepared using the Phase-Prep BAC DNA kit (Sigma-Aldrich) following the manufacturer's protocol. Fosmids used were G248P85689G4 (white, hg18 chr17:3143486531475400), G248P84883A8 (green, hg18 chr17:3146 8941-31505286) and G248P8961D8 (red, maps to hg18 twice at chr17:31537181-31574736 and chr17:3 1638770-31676303). The green clone was labelled with Dinitrophenol (DNP)-11-dUTP (PerkinElmer) and detected with rabbit anti-DNP and Alexa 488 conjugated goat anti-rabbit IgG. The red clone was labelled with Digoxigenin (DIG)-11-dUTP (Roche) and detected with monoclonal mouse anti-DIG IgG (Sigma-Aldrich) and Texas red conjugated donkey anti-mouse IgG (Invitrogen). The white clone was labelled with biotin16-dUTP and detected with one layer Cy3-avidin. After detection, slides were mounted with SlowFade Gold ${ }^{\circ}$ (Invitrogen) mounting solution containing 4', 6-diamidino-2-phenylindole (Invitrogen). Images were captured on a Zeiss Axioplan fluorescent microscope and processed with the SmartCapture software (Digital Scientific UK).

\section{Statistical analysis}

To analyse the effect of CCL3L1 on HIV load at initiation of HAART, we initially constructed a generalised linear model using SPSS 20.0 (IBM) and a gammaidentity link, as previously published. This link function did not model the data (which included new clinical data) well, and a gamma-log link provided a better fit to the data. Notably, for the previously-published $\beta$ defensin dataset [9], both gamma-identity and gammalog links model the data well, and although the gammaidentity model was chosen, the two models are almost indistinguishable based on several goodness-of-fit criteria and report very similar significance levels, therefore not calling our previous results into question. The model was calculated using type III sum of squares ANOVA, with goodness-of-fit analysed using Wald statistics.To examine the effect of CCL3L1 copy number on CD4+ count following initiation of HAART, we constructed a generalised linear mixed model, using STATA, where the dependent variable (CD4+ count) was modelled as a Gaussian distribution. In this model, we assigned 
population and disease status as fixed factors, initial CD4+ count and time since HAART initiation as scalar covariates and integer copy number as an ordinal covariate. The model was calculated using type III sum of squares ANOVA, with a variance correction to allow for multiple $\mathrm{CD} 4+$ timepoint readings from a single patient.

\section{Results}

\section{Analysis and validation of copy number typing}

We used a previously-published and well-established method for copy number typing, called the paralogue ratio test (PRT) to type CCL3L1 copy number (Figure 1a).

1134 samples were tested in total, and integer copy number called using a Gaussian mixture model approach (Figure 2, see Methods) after removal of one outlier with very high copy number ( 14). 192 (16.9\%) samples gave an integer copy number call posterior probability of less than 0.8 . Of these, 57 (30\%) had been tested in duplicate, of these 57 duplicates, 34 (60\%) supported the original copy number call and 23 supported the alternative copy number call (Additional 6: Figure S4). If we conservatively assume that there is no correlation between the samples selected for duplicate testing because of high coefficient of variation values and the samples giving posterior probability values $<0.8$, we can estimate the error rate to be $6.7 \%$, and these errors will involve an incorrect call of $+/-1$ copy number. Error rate is likely to be significantly lower than this because samples were selected for duplicate testing based on high coefficient of variation values, and are therefore likely to be significantly enriched for miscalled samples.

To validate our copy number calling at higher copy numbers, we used fibre-FISH on extended DNA fibres from cell lines derived from a parent-child trio from the YRI HapMap population. Our estimates, estimated from PRT prior to fibre-FISH analysis, agreed with the number of copies determined by FISH (Figure 1b). This is shown by the fosmid probe labelled red, which maps to the CCL3L1 repeat and has been used previously to estimate copy number in humans [35]. Interestingly, of the 14 CCL3L1 repeats directly visualised in these three trios, 11 have a repeat structure that includes a fosmid probe, labelled in green, which covers a region between the CCL4 gene and the TBC1D3 and CCL3L1 genes. This is in contrast to CCL3L1 repeats previously visualised in Europeans, where the repeat appears to be represented just by the red-labelled probe, and has been estimated to be $90 \mathrm{~kb}$ in size. Therefore, in Yoruba at least, and perhaps in other sub-Saharan Africans, there appears to be heterogeneity in CCL3L1 repeat structure not yet observed in Europeans.

Concordance of the three PRT assays is an important test of heterogeneity of the repeat. Studies of CCL3L1/ CCL4L1 copy number in European populations using the PRT method by us (unpublished data) and others [23] have shown concordance between all three probes, supporting the idea that this region is copy number variable en bloc and averaging the values of the three probes accurately reflects the copy number of this block. However, in this study, a small number of Ethiopian samples gave consistently high coefficient of variation scores due to one probe repeatedly giving discordant results. These can be seen as outliers on Additional file 4: Figure S2, and example data from samples are given in Additional file 7: Figure S5. This suggests either sub-Saharan African-specific copy number heterogeneity or rare duplication of the PRT reference locus, and is likely to contribute to the error rate observed in these data. All three PRT assays map to the region represented by the red fosmid probe in fibreFISH, and this PRT heterogeneity was not observed in the YRI or Tanzanian samples, strongly suggesting that this heterogeneity is of a different nature to that observed by fiber-FISH, and is perhaps confined to the Ethiopian population.

To further validate our CCL3L1 copy number calls, for the YRI HapMap samples we compared our estimates with arrayCGH data previously generated using the Agilent $210 \mathrm{~K} \mathrm{CNV}$ chip [6]. There is a clear positive correlation between the two methods, and it is also clear that the PRT generates data that clusters effectively into integer copy numbers, particularly at lower copy numbers, in contrast to aCGH where there is considerable overlap of copy number classes (Figure 1c).

\section{CCL3L1 copy number distribution in different populations} The copy number distributions are shown in Table 1. As has been observed previously, the copy number range for all three African populations is higher than European populations, where the common copy number range is between 1 and 4 copies per diploid genome [22]. Of the three African populations, the YRI show the highest mean copy number, although the Ethiopian population shows the greatest range (between $0-8$ copies) and one Tanzanian shows a particularly high copy number of 14 .

There is a small but marginally significant difference ( $p=0.02$, $t$-test) between the mean copy number of the Ethiopian HIV-only and the HIV-TB co-infected cohort, although this is not replicated in the smaller Tanzanian cohort $(\mathrm{p}=0.69)$. Indeed analysis by combining $\mathrm{CNV}$ calling and association testing using CNVtools, which can account for differential bias effects between cohorts, reported a non-significant effect for the Ethiopian dataset $(\mathrm{p}=0.52)$, suggesting a very subtle technical bias between the DNA plates containing 


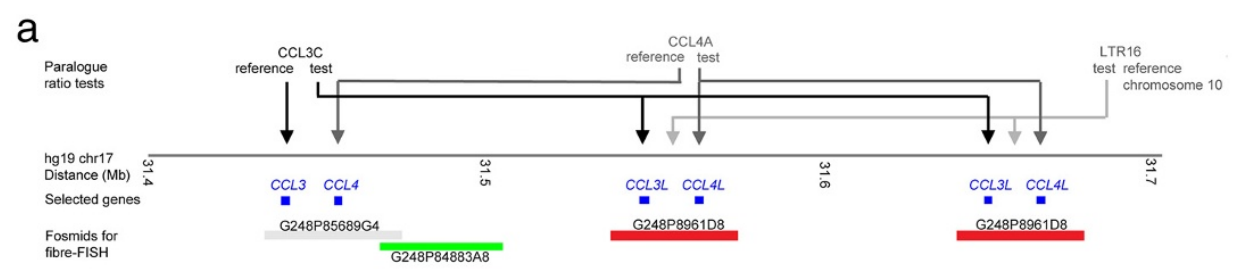

b
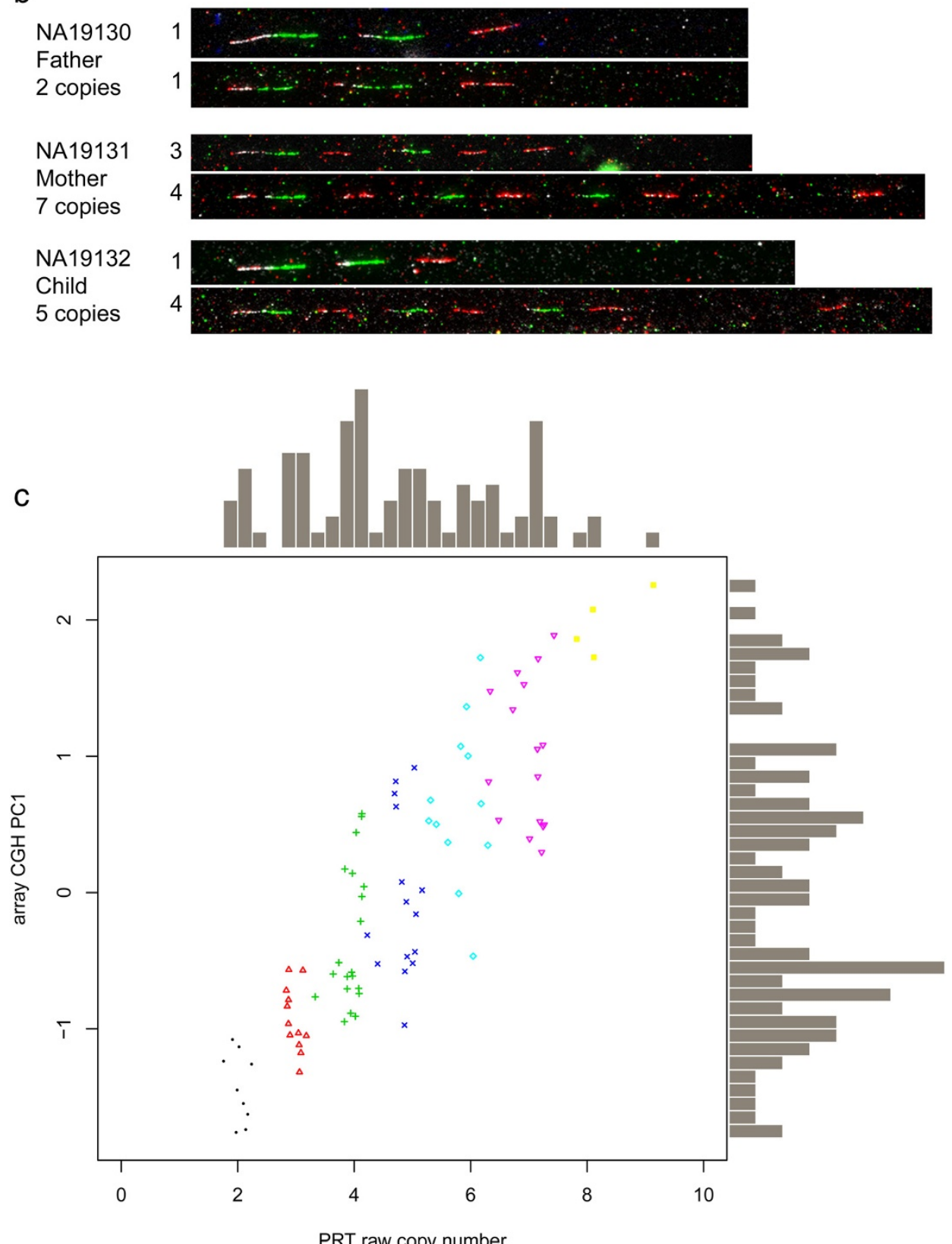

Symbol $\cdot \Delta+\times 0 \nabla$

$\begin{array}{llllllll}\text { Copy number } & 2 & 3 & 4 & 5 & 6 & 7 & 8\end{array}$

Figure 1 (See legend on next page.) 
(See figure on previous page.)

Figure 1 Validation of CNV using array CGH and fibre-FISH. a) The reference genomic region studied, showing the location of the sequences used in the paralogue ratio test (PRT) and the fosmids used for fibre-FISH analysis. b) Fibre-FISH analysis on stretched DNA fibres from three lymphoblastoid cell lines using the fosmid probes shown in part a). The three cell lines are from a YRI parent-child trio recruited for the HapMap project, with their DNA sample IDs given. PRT copy number estimates are given under each ID, and copy number estimated from each stretched individual chromosome given immediately to the left of a representative fiber-FISH image. c) Comparison of raw PRT estimates of CCL3L1 copy number on HapMap YRI samples ( $x$-axis) with estimates from arrayCGH data (y-axis). Points are coloured according to final integer copy number estimates, as indicated by the key below the scatterplot. PC1=first principal component of arrayCGH data.

HIV-only samples and those containing HIV-TB samples.

\section{Association of copy number with clinical parameters}

To investigate the effect of CCL3L1 copy number on viral load, immediately prior to HAART, we fitted a generalised linear model to the data, with population of origin, tuberculoisis co-infection status and CD4+ count immediately prior to HAART as cofactors. We found significant association with population of origin, TB infection and CD4+ count, but no effect of CCL3L1 copy number (Table 2). We repeated the analysis using raw copy number values, with no change.

To investigate the effect of CCL3L1 copy number on immune reconstitution following HAART, we measured CD4+ count at 12, 24, 36 and 48 week intervals following initiation of treatment. Using a multivariate linear mixed effects model to control for the multiple repeated measurements contributed by the same patient at different timepoints, we found a significant association of time since initiation of treatment, CD4+ levels at initiation of treatment, population of origin, TB co-infection status and CCL3L1 copy number $(\mathrm{p}=0.012$, Table 3 , Figure 3). The direction of effect of CCL3L1 copy number, with higher copy number associated with poorer immune reconstitution, agrees with previous studies [12].

\section{Discussion}

It has been observed previously that, despite HAART being effective at reducing HIV load to below measurable levels, CD4+ cell count does not always return to healthy levels [36]. This might be due to a variety of factors, including host genetics and co-infection status. Indeed, we demonstrate in this study (Table 3) that both initial baseline CD4+ cell count and absence of TB have a positive effect on the CD4+ count following initiation of HAART, a commonly used measure of immune reconstitution. The role of host genetic variation in influencing different rates of immune reconstitution during HAART is not well understood, yet is of increasing importance as HAART programmes are initiated and continued in areas of high HIV prevalence. Several candidate genes have been suggested to play a role, including a haplotype of the TRAIL gene and copy number variation of the $\beta$-defensin genes $[9,37]$. This study suggests that CCL3L1 copy number has a stronger effect on immune reconstitution than $\beta$-defensins $(\beta$-defensin $\beta=-3.63$ CD $4+$ cells $/ \mathrm{ml}$ per copy, CCL3L1 $\beta=-4.75$ CD $4+$ cells $/ \mathrm{ml}$

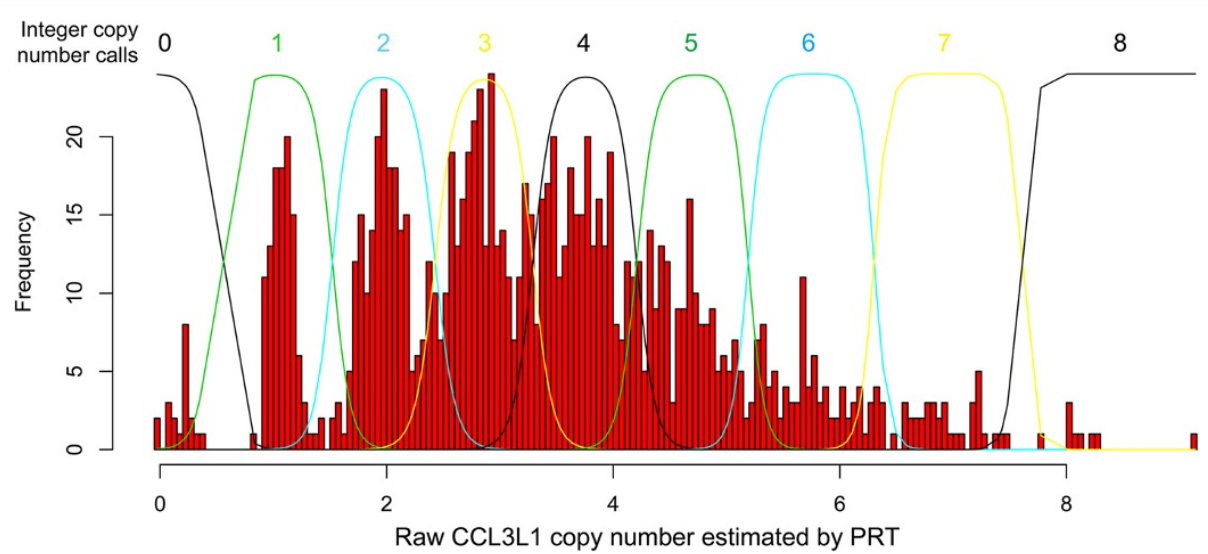

Figure 2 Calling integer CCL3L1 copy number from raw PRT data. The histogram shows the copy number distribution of 1133 individuals. A Gaussian mixture model, with seven components, is fitted to the data, and each individual component is plotted with the corresponding integer copy number shown above each peak. 
Table 1 CCL3L1 Copy number distribution and comparisons between populations

\begin{tabular}{|c|c|c|c|c|c|c|c|c|c|c|}
\hline \multirow{2}{*}{$\frac{C C L 3 L 1 \text { copy number }}{0}$} & \multicolumn{2}{|c|}{ Tanzanian HIV } & \multicolumn{2}{|c|}{ Tanzanian HIV + TB } & \multicolumn{2}{|c|}{ Ethiopian HIV } & \multicolumn{2}{|c|}{ Ethiopian HIV + TB } & \multicolumn{2}{|c|}{ YRI unrelated } \\
\hline & 0 & 0 & 0 & 0 & 7 & 0.03 & 11 & 0.03 & 0 & 0 \\
\hline 1 & 2 & 0.01 & 2 & 0.01 & 40 & 0.17 & 46 & 0.14 & 7 & 0.12 \\
\hline 2 & 25 & 0.12 & 13 & 0.09 & 39 & 0.17 & 71 & 0.22 & 8 & 0.14 \\
\hline 3 & 52 & 0.25 & 40 & 0.28 & 41 & 0.17 & 86 & 0.26 & 13 & 0.22 \\
\hline 4 & 56 & 0.27 & 48 & 0.33 & 42 & 0.18 & 55 & 0.17 & 10 & 0.17 \\
\hline 5 & 43 & 0.21 & 22 & 0.15 & 33 & 0.14 & 35 & 0.11 & 9 & 0.16 \\
\hline 6 & 22 & 0.11 & 15 & 0.10 & 21 & 0.09 & 17 & 0.05 & 8 & 0.14 \\
\hline 7 & 4 & 0.02 & 5 & 0.03 & 8 & 0.03 & 5 & 0.02 & 3 & 0.05 \\
\hline 8 & 1 & 0 & 0 & 0 & 4 & 0.02 & 0 & 0 & 0 & 0 \\
\hline 9 & 0 & 0 & 0 & 0 & 0 & 0 & 0 & 0 & 0 & 0 \\
\hline 10 & 0 & 0 & 0 & 0 & 0 & 0 & 0 & 0 & 0 & 0 \\
\hline 14 & 1 & 0 & 0 & 0 & 0 & 0 & 0 & 0 & 0 & 0 \\
\hline N & \multicolumn{2}{|c|}{206} & \multicolumn{2}{|c|}{145} & \multicolumn{2}{|c|}{235} & \multicolumn{2}{|c|}{326} & \multicolumn{2}{|c|}{58} \\
\hline mean & \multicolumn{2}{|c|}{4.024} & \multicolumn{2}{|c|}{3.966} & \multicolumn{2}{|c|}{3.353} & \multicolumn{2}{|c|}{3.000} & \multicolumn{2}{|c|}{4.72} \\
\hline
\end{tabular}

per copy). However, unlike $\beta$-defensin copy number, we find no effect of CCL3L1 copy number on viral load during acute HIV infection, just prior to initiation of HAART.

Previous studies have used combined data from different ethnic groups, with very different CCL3L1 copy numbers, with HAART started at different CD4 count thresholds. It might be argued that variation in ethnicity was a confounding factor, so that ethnicity rather than CCL3L1 copy number per se, was responsible for the variation in immunological reconstitution. While in no way a genetically homogeneous cohort, a fact that we attempt to account for in part by using country of origin as a cofactor in our analyses, our study does not combine two dichotomous ethnic groups with very different CCL3L1 copy number counts and different levels of access to healthcare [12]. Our entire cohort is also completely naïve to antiretroviral therapy prior to initiation of HAART, unlike those previously studied $[12,38]$.

Although we have taken care to ensure the optimum quality of our copy number typing, problems remain particularly in distinguishing higher copy numbers,

Table 2 Model fitting - output viral load

\begin{tabular}{lll}
\hline Model & $\begin{array}{l}\beta \text { coefficient }(\mathbf{9 5 \%} \mathrm{Cl}) \\
\text { (copies } / \mathbf{m L})\end{array}$ & P value \\
\hline Population & $-0.67(-0.97,-0.36)$ & $<0.001$ \\
No TB Co-infection & $-0.42(-0.69,-0.16)$ & 0.002 \\
CD4+ count (cells $\left./ \mathrm{mm}^{3}\right)$ & $-0.003(-0.006,-0.001)$ & 0.008 \\
CCL3L1 copy number & $-0.068(-1.45,0.009)$ & 0.084 \\
\hline
\end{tabular}

( $n=656$ observations). which are frequent in sub-Saharan African populations. Part of this is technical, due to inherent noise in the assays used, and part biological, due to the variation in repeat structure apparent in certain populations. Both issues cannot be resolved easily without more extensive work on the nature and extent of structural variation at this locus in different populations, and we suggest that this should be a prerequisite before a comprehensive analysis of the clinical role of CCL3L1 copy number can be made. The Genome Reference Consortium has assembled a reference allele from sequencing BACs from a genomic library derived from a hydatidaform mole, which contains one copy of the CCL3L1 and CCLAL1 genes and is likely to represent the most common allele in Europeans (accession number GL383560.1). However we show here that the high-copy alleles characteristic of African populations are not necessarily simply related to the European alleles, and there is clearly a need for accessible physical remapping approaches that can be applied to a

Table 3 Model fitting - output CD4 count after HAART

\begin{tabular}{lll}
\hline Model & $\begin{array}{l}\boldsymbol{\beta} \text { coefficient } \\
\left(\text { cells } / \mathbf{m m}^{3}\right)\end{array}$ & P value \\
\hline Time after HAART (weeks) & $2.61(2.37,2.85)$ & $<0.001$ \\
Baseline CD4+ (cells/mm $\left.{ }^{3}\right)$ & $0.88(0.77,1.00)$ & $<0.001$ \\
Population & $19.36(5.08,33.63)$ & 0.008 \\
No TB co-infection & $16.64(3.33,29.95)$ & 0.014 \\
CCL3L1 copy number & $-4.75(-8.46,-1.05)$ & 0.012 \\
\hline
\end{tabular}

$\mathrm{N}=1692$ observations on 491 patients, mixed effects model accounting for repeat measures at different timepoints. 


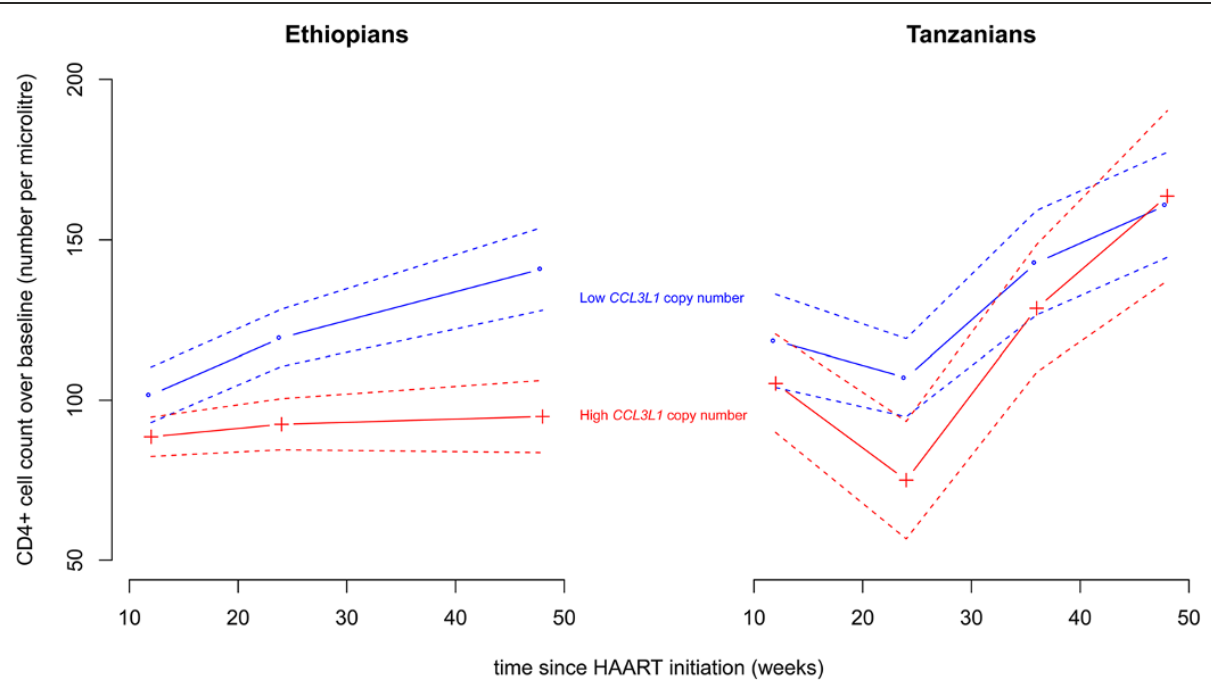

Figure 3 Response to HAART in Ethiopians and Tanzanians stratified by CCL3L1 copy number. Average values (solid line) and standard error of the mean (dashed line) of CD4+ cell counts was calculated for the different timepoints following initiation of HAART. Patients were stratified according to CCL3L1 copy number, with high copy number being greater than the median integer value, which was 3 copies in Ethiopians and 4 copies in Tanzanians. $N=798$ CD4+ values in Ethiopians, $n=894$ CD4+ values in Tanzanians.

significant number of samples to fully characterise structural variation at this locus.

There are three other caveats in interpretation of our study. Firstly, although we control for co-infection with tuberculosis, which represents the major co-morbidity in these populations, we cannot rule out that the effect of $C C L 3 L 1$ copy number is indirect, via another infection, rather directly on immune reconstitution. Secondly, as stated previously, the copy number variation involves the genes for the chemokine CCL4L1, and TBC1D3, a protein involved in macropinocytosis [39]. Although CCL3L1 is the favoured candidate for mediating the effect of copy number based on the known functional role of the chemokine, a role for the other gene products should not be completely ruled out. Thirdly, we also cannot rule out an indirect effect of CCL3L1 copy number mediated by an effect on CD4+ levels immediately after seroconversion, which have been shown to affect immune reconstitution [40].

\section{Conclusions}

Taken together, our data support a role for CCL3L1 copy number in the immune reconstitution following initiation of HAART to treat HIV infection. These data also support the suggestion that treatment of HIV using MIP1 $\alpha$ analogues as part of a combined HIV treatment regimen, might adversely affect immune reconstitution, but a small molecule that interferes with MIP1 $\alpha$ interactions with cognate receptors might aid immune reconstitution.

\section{Additional files}

Additional file 1: Table S1. Sample sizes used in the study. Arm 3 was recruited with CD4 > 200 and TB, had CCL3L1 copy number for 96 patients called but was not matched to clinical data for this study.

Additional file 2: Table S2. Baseline characteristics of patients analysed. Additional file 3: Figure S1. Analysis of PRT measurement noise in control samples. Individual unrounded PRT values are plotted on the $y$ axis, according to the different copy numbers of the four controls ( $x$-axis). Each point is coloured according which of the three different PRT assays generated it, all three assays measuring CCL3L1 copy number.

Additional file 4: Figure S2. Clustering of PRT raw data between different assays. For the complete dataset $(n=1133)$, density scatterplots were draw comparing each of the three different assays with each other. Axis labels indicate raw PRT values, and the colour bar on the left indicates the density of individual datapoints. One extreme point has been omitted.

Additional file 5: Figure S3. Analysis of the distribution of PRT values about a single copy number. a). The density of raw unrounded PRT values of the control samples, shown in supplementary Figure 1, is plotted, with values normalised to centre on a mean of zero. The red dotted line represents a Gaussian distribution with a mean and standard deviation taken from the PRT data. The blue dashed line represents a Gaussian distribution fitted to the PRT data. b). Gaussian quantile-quantile plot of raw unrounded PRT values of the control samples. Each value is plotted according the copy number of the control sample, as shown in the legend. The straight line is plotted through the first and third quantiles.

Additional file 6: Figure S4. Confidence of integer copy number calls from raw PRT data.Raw PRT calls of the entire dataset (average of three PRT assays) are plotted on the $x$-axis with posterior probability of the resulting integer copy number call on the $y$-axis. Points plotted as red triangles are those where $P<0.8$ with a repeat measurement which gave a different estimate of integer copy number $( \pm 1)$. Points plotted as green crosses are those where $P<0.8$ with a repeat measurement which gave the same estimate of integer copy number.

Additional file 7: Figure S5. Examples of assay heterogeneity. Six Ethiopian samples are highlighted, together with the raw PRT ratios, coloured by PRT assay, after several repeat tests. 


\section{Competing interests}

EJH has received grant funding from Pfizer Inc, which had no influence in the conception, design or analysis of this work, and no role in manuscript preparation or publication.

\section{Authors' contributions}

$\mathrm{EA}$ and $\mathrm{EJH}$ concieved and designed the study. Experiments were performed by $\mathrm{LOH}, \mathrm{JB}, \mathrm{RH}, \mathrm{BF}$ and FY. Data were analysed by EJH, LOH, JB, BF, FY and MV. Clinical data and patient samples were provided by EA, AH, EN, GY, WA, SM, OM, EM, MJ, FM and GA. All authors read and approved the final manuscript.

\section{Acknowledgements}

Thanks to Don Conrad for access to the Agilent $210 \mathrm{k}$ arrayCGH data, Mark Jobling for access to ABI3130xI capillary electrophoresis platform, and the patients for participation in this study.

\section{Funding}

This work was supported by a United Kingdom Medical Research Council New Investigator award [grant number GO801123] and a Wellcome Trust project grant [WT087663] to E.J.H.; European \& Developing Countries Clinical Trials Partnership [grant numbers CT.2005.32030.001, CG_TA.05.40204_005]; and the Swedish International Development Cooperation Agency/ Department for Research Cooperation [grant numbers HIV-2006-031, SWE 2007-270, VR 521-2011-3437]. Core facility funding was supported by the Wellcome Trust [grant number WT098051].

\section{Author details}

'Department of Clinical Pharmacology, Karolinska Institutet, Stockholm, Sweden. ${ }^{2}$ Department of Genetics, University of Leicester, University Road, LE1 7RH, Leicester, UK. ${ }^{3}$ Department of Pharmacology, Addis Ababa University, Addis Ababa, Ethiopia. ${ }^{4}$ Unit of Pharmacology, School of Pharmacy, Muhimbili University of Health and Allied Sciences, Dar es Salaam, Tanzania. ${ }^{5}$ Internal Medicine, Addis Ababa University, Addis Ababa, Ethiopia. ${ }^{6}$ Institution of Medicine, Unit of Infectious Diseases, Karolinska Institutet, Karolinska University Hospital, Huddinge, Sweden. ${ }^{7}$ Department of Internal Medicine, Muhimbili National Hospital, Dar es Salaam, Tanzania. ${ }^{8}$ Department of Internal Medicine, Muhimbili University of Health and Allied Sciences, Dar es Salaam, Tanzania. ' Wellcome Trust Sanger Institute, Hinxton, Cambridge, UK. ${ }^{10}$ College of Medicine, Biological Sciences and Psychology, University of Leicester, Leicester, UK

Received: 29 May 2013 Accepted: 28 October 2013

Published: 12 November 2013

\section{References}

1. Mathers CD, Loncar D: Projections of global mortality and burden of disease from 2002 to 2030. PLoS Medicine 2006, 3(11):e442.

2. An P, Winkler CA: Host genes associated with HIV/AIDS: advances in gene discovery. Trends in Genetics 2010, 26(3):119-131.

3. Petrovski S, Fellay J, Shianna KV, Carpenetti N, Kumwenda J, Kamanga G, Kamwendo DD, Letvin NL, McMichael AJ, Haynes BF, et al: Common human genetic variants and HIV-1 susceptibility: a genome-wide survey in a homogeneous African population. AIDS 2011, 25(4):513-518.

4. Locke DP, Sharp AJ, McCarroll SA, McGrath SD, Newman TL, Cheng Z, Schwartz S, Albertson DG, Pinkel D, Altshuler DM, et al: Linkage disequilibrium and heritability of copy-number polymorphisms within duplicated regions of the human genome. American Journal of Human Genetics 2006, 79(2):275-290.

5. Wain LV, Armour JAL, Tobin MD: Genomic copy number variation, human health, and disease. Lancet 2009, 374(9686):340-350.

6. Conrad DF, Pinto D, Redon R, Feuk L, Gokcumen O, Zhang Y, Aerts J, Andrews TD, Barnes C, Campbell P, et al: Origins and functional impact of copy number variation in the human genome. Nature 2009, 464(7289):704-712.

7. Stranger BE, Forrest MS, Dunning M, Ingle CE, Beazley C, Thorne N, Redon R, Bird CP, De Grassi A, Lee C, et al: Relative impact of nucleotide and copy number variation on gene expression phenotypes. Science 2007, 315(5813):848-853.

8. Pelak K, Need AC, Fellay J, Shianna KV, Feng S, Urban TJ, Ge D, De Luca A, Martinez-Picado J, Wolinsky SM, et al: Copy Number Variation of KIR Genes Influences HIV-1 Control. PLoS Biology 2011, 9(11):e1001208.
9. Hardwick RJ, Amogne W, Mugusi S, Yimer G, Ngaimisi E, Habtewold A, Minzi O, Makonnen E, Janabi M, Machado LR, et al: $\beta$-defensin Genomic Copy Number Is Associated With HIV Load and Immune Reconstitution in Sub-Saharan Africans. J Infect Dis 2012, 206(7):1012-1019.

10. Townson JR, Barcellos LF, Nibbs RJ: Gene copy number regulates the production of the human chemokine CCL3-L1. European Journal of Immunology 2002, 32(10):3016-3026.

11. Gonzalez E, Kulkarni H, Bolivar H, Mangano A, Sanchez R, Catano G, Nibbs RJ, Freedman BI, Quinones MP, Bamshad MJ, et al: The influence of CCL3L1 gene-containing segmental duplications on HIV-1/AIDS susceptibility. Science Signalling 2005, 307(5714):1434.

12. Ahuja SK, Kulkarni H, Catano G, Agan BK, Camargo JF, He W, O'Connell RJ, Marconi VC, Delmar J, Eron J, et al: CCL3L1-CCR5 genotype influences durability of immune recovery during antiretroviral therapy of HIV-1-infected individuals. Nature Medicine 2008, 14(4):413-420.

13. Menten P, Struyf S, Schutyser E, Wuyts A, De Clercq E, Schols D, Proost P, Van Damme J: The LD78 $\beta$ isoform of MIP-1a is the most potent CCR5 agonist and HIV-1-inhibiting chemokine. J Clin Investig 1999, 104(4):R1.

14. Carpenter D, Mclntosh R, Pleass R, Armour J: Functional effects of CCL3L1 copy number. Genes Immun 2012, 13(5):374-379.

15. Picton AC, Paximadis M, Tiemessen CT: Contribution of variable CCL3L copy number to CCL3 protein production in two ethnically divergent South African populations. Infect Genet Evol 2013, 14:347-356.

16. Proost P, Menten P, Struyf S, Schutyser E, De Meester I, Van Damme J: Cleavage by CD26/dipeptidyl peptidase IV converts the chemokine LD78 $\beta$ into a most efficient monocyte attractant and CCR1 agonist. Blood 2000, 96(5):1674-1680.

17. Menten P, Wuyts A, Van Damme J: Macrophage inflammatory protein-1. Cytokine \& Growth Factor Reviews 2002, 13(6):455-481.

18. Liu S, Yao L, Ding D, Zhu H: CCL3L1 copy number variation and susceptibility to HIV-1 infection: a meta-analysis. PLOS ONE 2010, 5(12):e15778.

19. Cantsilieris S, White SJ: Correlating multiallelic copy number polymorphisms with disease susceptibility. Hum Mutat 2012, 34:1-13.

20. Shrestha S, Nyaku M, Edberg JC: Variations in CCL3L gene cluster sequence and non-specific gene copy numbers. BMC Research Notes 2010, 3(1):74.

21. Field SF, Howson JM, Maier LM, Walker S, Walker NM, Smyth DJ, Armour JA, Clayton DG, Todd JA: Experimental aspects of copy number variant assays at CCL3L1. Nat Med 2009, 15(10):1115-1117.

22. Carpenter D, Walker S, Prescott N, Schalkwijk J, Armour JA: Accuracy and differential bias in copy number measurement of CCL3L1 in association studies with three auto-immune disorders. BMC Genomics 2011, 12(1):418.

23. Walker S, Janyakhantikul S, Armour JA: Multiplex Paralogue Ratio Tests for accurate measurement of multiallelic CNVs. Genomics 2009, 93(1):98-103.

24. Mamtani M, Mummidi S, Ramsuran V, Pham M-H, Maldonado R, Begum K, Valera MS, Sanchez R, Castiblanco J, Kulkarni H, et al: Influence of variations in CCL3L1 and CCR5 on tuberculosis in a northwestern Colombian population. J Infect Dis 2011, 203(11):1590-1594.

25. Li H, Xie H-Y, Zhou L, Wang W-L, Liang T-B, Zhang M, Zheng S-S: Polymorphisms of CCL3L1/CCR5 genes and recurrence of hepatitis $B$ in liver transplant recipients. Hepatobiliany Pancreat Dis Int 2011, 10(6):593-598.

26. Grünhage F, Nattermann J, Gressner OA, Wasmuth HE, Hellerbrand C, Sauerbruch T, Spengler U, Lammert F: Lower copy numbers of the chemokine CCL3L1 gene in patients with chronic hepatitis C. Journal of Hepatology 2010, 52(2):153-159

27. Mamtani M, Matsubara T, Shimizu C, Furukawa S, Akagi T, Onouchi Y, Hata A, Fujino A, He W, Ahuja SK: Association of CCR2-CCR5 haplotypes and CCL3L1 copy number with Kawasaki disease, coronary artery lesions, and IVIG responses in Japanese children. PLOS ONE 2010, 5(7):e11458.

28. Kim H-E, Kim J-J, Han MK, Lee K-Y, Song MS, Lee H-D, Kim DS, Yu JJ, Park I-S, Yun SW, et al: Variations in the Number of CCL3L1 Gene Copies and Kawasaki Disease in Korean Children. Pediatr Cardiol 2012, 1:5.

29. Carpenter D, Färnert A, Rooth I, Armour JA, Shaw M-A: CCL3L1 copy number and susceptibility to malaria. Infect Genet Evol 2012, 12:1147-1154.

30. Larsen MH, Thørner LW, Zinyama R, Amstrup J, Kallestrup P, Gerstoft J, Gomo E, Erikstrup C, Ullum H: CCL3L gene copy number and survival in an HIV-1 infected Zimbabwean population. Infect Genet Evol 2012, 12:1087-1093

31. Kuhn L, Schramm DB, Donninger S, Meddows-Taylor S, Coovadia AH, Sherman GG, Gray GE, Tiemessen CT: African infants' CCL3 gene copies influence perinatal HIV transmission in the absence of maternal nevirapine. AIDS 2007, 21(13):1753-1761. 
32. Yimer G, Ueda N, Habtewold A, Amogne W, Suda A, Riedel K-D, Burhenne J, Aderaye G, Lindquist L, Makonnen E: Pharmacogenetic \& pharmacokinetic biomarker for efavirenz based ARV and rifampicin based anti-TB drug induced liver injury in TB-HIV infected patients. PLOS ONE 2011, 6(12):e27810.

33. Mugusi SF, Ngaimisi E, Janabi MY, Mugusi FM, Minzi OM, Sasi PG, Bakari M, Lindquist L, Aklillu E, Sandstrom EG: Risk factors for mortality among HIVpositive patients with and without active tuberculosis in Dar es Salaam, Tanzania. Antiviral Therapy 2012, 17(2):265-274.

34. Barnes C, Plagnol V, Fitzgerald T, Redon R, Marchini J, Clayton D, Hurles ME: A robust statistical method for case-control association testing with copy number variation. Nat Genet 2008, 40(10):1245-1252.

35. Perry GH, Yang F, Marques-Bonet T, Murphy C, Fitzgerald T, Lee AS, Hyland C, Stone AC, Hurles ME, Tyler-Smith C, et al: Copy number variation and evolution in humans and chimpanzees. Genome Res 2008, 18(11):1698-1710.

36. Corbeau P, Reynes J: Immune reconstitution under antiretroviral therapy: the new challenge in HIV-1 infection. Blood 2011, 117(21):5582-5590

37. Haas DW, Geraghty DE, Andersen J, Mar J, Motsinger AA, Richard T, Unutmaz D, Benson CA, Ritchie MD, Landay A, et al: Immunogenetics of CD4 lymphocyte count recovery during antiretroviral therapy: an AIDS Clinical Trials Group study. J Infect Dis 2006, 194(8):1098-1107.

38. Bhattacharya T, Stanton J, Kim E-Y, Kunstman KJ, Phair JP, Jacobson LP, Wolinsky SM: CCI3I1 and hiv/aids susceptibility. Nature Medicine 2009, 15(10):1112-1115.

39. Frittoli E, Palamidessi A, Pizzigoni A, Lanzetti L, Garrè M, Troglio F, Troilo A, Fukuda M, Di Fiore PP, Scita G, et al: The primate-specific protein TBC1D3 is required for optimal macropinocytosis in a novel ARF6-dependent pathway. Molecular Biology of the Cell 2008, 19(4):1304-1316.

40. Kulkarni H, Okulizz JF, Grandits G, Crum-Cianflone NF, Landrum ML, Hale B, Wortmann G, Tramont E, Polis M, Dolan M: Early Postseroconversion CD4 Cell Counts Independently Predict CD4 Cell Count Recovery in HIV-1-Postive Subjects Receiving Antiretroviral Therapy. JAIDS Journal of Acquired Immune Deficiency Syndromes 2011, 57(5):387-395.

doi:10.1186/1471-2334-13-536

Cite this article as: Aklillu et al: CCL3L1 copy number, HIV load, and immune reconstitution in sub-Saharan Africans. BMC Infectious Diseases 2013 13:536

\section{Submit your next manuscript to BioMed Central and take full advantage of:}

- Convenient online submission

- Thorough peer review

- No space constraints or color figure charges

- Immediate publication on acceptance

- Inclusion in PubMed, CAS, Scopus and Google Scholar

- Research which is freely available for redistribution 